Page 1 of 20

An examination of the concordance between self-reported collisions, driver records, and insurance claims in older drivers

\author{
Michelle M. Porter \\ Journal of Safety Research
}

67: 211-215, 2018

https://doi.org/10.1016/j.jsr.2018.07.007 
Page 2 of 20

\title{
An examination of the concordance between self-reported collisions, driver records, and insurance claims in older drivers
}

\begin{abstract}
Background: Previous studies of older drivers have found that there are discrepancies between their retrospective self-reports of collisions and the official jurisdictional record. Objectives: The purpose of this study was to examine how older drivers self-report collisions in comparison to what was recorded in their official driver abstract as well as insurance claims, in a prospective study. Methods: Participants ( $n=125$, age $\geq 70$ years) in this study were part of the University of Manitoba site of the Candrive longitudinal study of older drivers. During the operation of the Manitoba site (2009 to 2013), participants were periodically asked to report on any collisions (at-fault or not) in which they were involved, while they were enrolled in the study. In addition, driver records (abstracts and insurance claims) from the provincial licensing agency and public insurer (Manitoba Pubic Insurance; MPI) were provided annually. Results: In total there were 101 separate instances of collisions (regardless of atfault status), whether self-reported, or recorded by MPI. There were 20 at-fault collisions that were recorded on the driver abstract. Eighteen of these collisions were self-reported by participants. In total, our participants were involved in 70 insurance claims (42 at-fault) -61 of these were self-reported to study staff. In addition, there were 31 collisions that were self-reported to study staff, that were not reported to MPI. Conclusions: In this prospective study, older drivers were diligent in reporting collisions in which they were involved. While some collisions were not reported that ultimately became a claim or part of their driver abstract, the biggest discrepancy was in the collisions that were reported to study staff but that were not reported to authorities.
\end{abstract}

Keywords: Automobile driving, road safety, motor vehicle collisions, aging 
Page 3 of 20

\section{Introduction}

The primary outcome variable in many studies related to road safety is the motor vehicle collision (MVC). Different studies use different sources of MVC data though, and this has implications for the results and the interpretation of those results (Arthur et al., 2005). Collisions self-reported by study participants are easily attained with little cost or time using questionnaires. However, there are many possible issues associated with self-report data. These include cognitive and communicative biases such as question confusion and selective memory (Arthur et al., 2005). As af Wåhlberg and Dorn (2015) state "if specific information is not readily accessible in memory respondents may draw from their belief about their standing in the general domain", in other words "reflect self-presentational needs" or underreport collisions they have been in.

For these reasons, many adopt the use of jurisdictional records as their source of MVC data. Jurisdictional records are also susceptible to validity issues though. These include: missing records (McGuire 1973), biases in whose collisions are recorded (McGuire, 1973), as well as many interjurisdictional and police/staff issues that result in inaccurate records (Zylman, 1972).

As many jurisdictions around the world prepare for the growing older driver population, much research is being undertaken to understand the road safety issues of older drivers, and in particular to determine which health and/or aging factors might be implicated in increasing crash risks. In light of this, having a proper outcome variable is critical, as research has shown that the type of MVC data can affect the results (Smith, 1976; Arthur, 2005). In older drivers, a few studies have directly compared self-report and jurisdictional data. These will be discussed below. 
Page 4 of 20

Owsley et al. (1991) asked 53 older drivers to report on "police-reported accidents" in the prior five year period. They found that 12 of 18 individuals who had state-recorded MVCs, did not report those MVCs. Ball et al. (1993) also found that a large number of older drivers did not report MVCs that were recorded by the state. In this study of 294 older drivers, $67 \%$ of whom were involved in an MVC in the previous five years, only $27 \%$ self-reported the same number of MVCs, as were found in the state record.

Marottoli et al. (1997) also compared self-reported and state recorded MVCs, in the previous year, in a sample of 358 older drivers. In this study there were more drivers self-reporting collisions, $\mathrm{n}=\mathbf{2 9}$, as compared to 13 state recorded collisions. Four individuals had a state recorded MVC, but did not selfreport it. Although not restricted to older drivers, Finestone et al. (2011) found that drivers who had survived a stroke, also had many discrepancies between official transportation department MVCs/conviction records and self-reports, for the previous 5 years. The levels of agreement according to kappa coefficients were moderate for both collisions and convictions, and using both sources of data resulted in $56 \%$ more collisions and $46 \%$ more convictions.

The most comprehensive study of the agreement between self-reported and state recorded MVC was conducted by McGwin et al. (1998). Older drivers were "asked how many accidents they had had over the past 5 years where the police were called to the scene...wherein they were the driver" (McGwin et al., 1998, pg 784). In the past 5 years, 175 had MVCs recorded by the state that met this definition. For 111 there was agreement between self-report and state records. A relatively large number $(n=64)$ did not report a crash that was recorded by the state, but only 14 reported a crash that was not recorded by the state. In this study they also went further and examined the prevalence of visual and cognitive impairments in those who had crashes, either both self-report, state recorded or both, as compared to 
those who did not crash. The definition of a crash did have an effect on their results such that when selfreported MVCs were used, only useful field of view was significant. When only state recorded collisions were used, or the combination of both types of collisions, then glaucoma also became significant. These authors concluded that "it is clear that caution is necessary when using self-report for the identification of crash-involved older drivers".

The one drawback with all of the older driver studies mentioned above is that they used retrospective data. In part this was done to ensure that the number of collisions was large enough for the statistical procedures used (Ball et al., 1993). Naturally, such retrospective designs have limitations in terms of memory (Arthur et al., 2005).

Anstey et al., studied older drivers both retrospectively (past 5 years) and prospectively for collisions. Prospectively they asked participants to use a monthly injury diary to record all crashes they might have been in while driving in the past month. If participants did not submit an injury diary there was followup, using the mail and phone calls. This study found that participants self-reported many more crashes than were recorded by state transport authorities, whether the data were retrospective or prospective.

Most recently Singletary et al. (2017), conducted a prospective trial in drivers 70 years of age and older, who were asked to recall collisions over the past year, specifically where police were called to the scene. This study found that there was substantial agreement between self-reported and state recorded collisions. 
Page 6 of 20

A synopsis of this literature shows that results vary quite substantially between studies in the level and direction of concordance. Factors that might affect these results include the country of study, the length of followup, and the types of collisions. Also, the sources of official collision data have been limited to government authorities. We are not aware of studies that have examined self-reported collisions versus insurance claims in older drivers. In a population level study, police-reported collisions were found to have missing information as compared to insurance records (Mills et al., 2011).

The purpose of this study was to examine the concordance between self-reported collisions, jurisdictionally recorded traffic crashes, as well as insurance claims, in a prospective study of older drivers. It was hypothesized that drivers would self-report more collisions than would become part of their driver record or an insurance claim.

\section{Methods}

\subsection{Design}

Data for this study came from a prospective longitudinal study of older drivers from seven sites in Canada (see Marshall et al, 2013 for details of the protocol). Volunteers who were recruited to participate in the study were generally found to be representative of older Canadian drivers (Gagnon et al., 2013). For the purposes of this investigation, participants from the Manitoba site were selected because insurance claim data were available from Manitoba Public Insurance (MPI), which acts as both the licensing authority, as well as the insurer for all personal vehicles in Manitoba. Other Candrive sites did not collect insurance claim data. 
For the Manitoba site, enrollment into the study began in August 2009 and ended in September 2010. Participants were followed until September 2013. All participants provided consent to the release of their driving record from MPI. Periodically during the study, participants were also asked to report on any collisions they were in.

\subsection{Participants}

In order to be eligible for the study, amongst several inclusion criteria (Marshall et al., 2013), older drivers had to be 70 years or older, drive at least 4 times per week, and reside in or near Winnipeg for at least 10 months of the year. The convenience sample was recruited using a variety of strategies (e.g., newspaper articles and other media, direct communications with retiree groups, and posters in community locations). Enrollment and retention details for all the Manitoba participants can be seen in Figure 1. At baseline, participants ( $n=125$; predetermined recruitment target number for Candrive) ranged in age from 70 to 89 years (mean $=75.3, S D=4.8$ ), with $69.6 \%$ being male.

All participants provided written informed consent to participate in the study which was approved by the University Ethics Board. This consent included a specific provision to receive driver and claim information from MPI. 


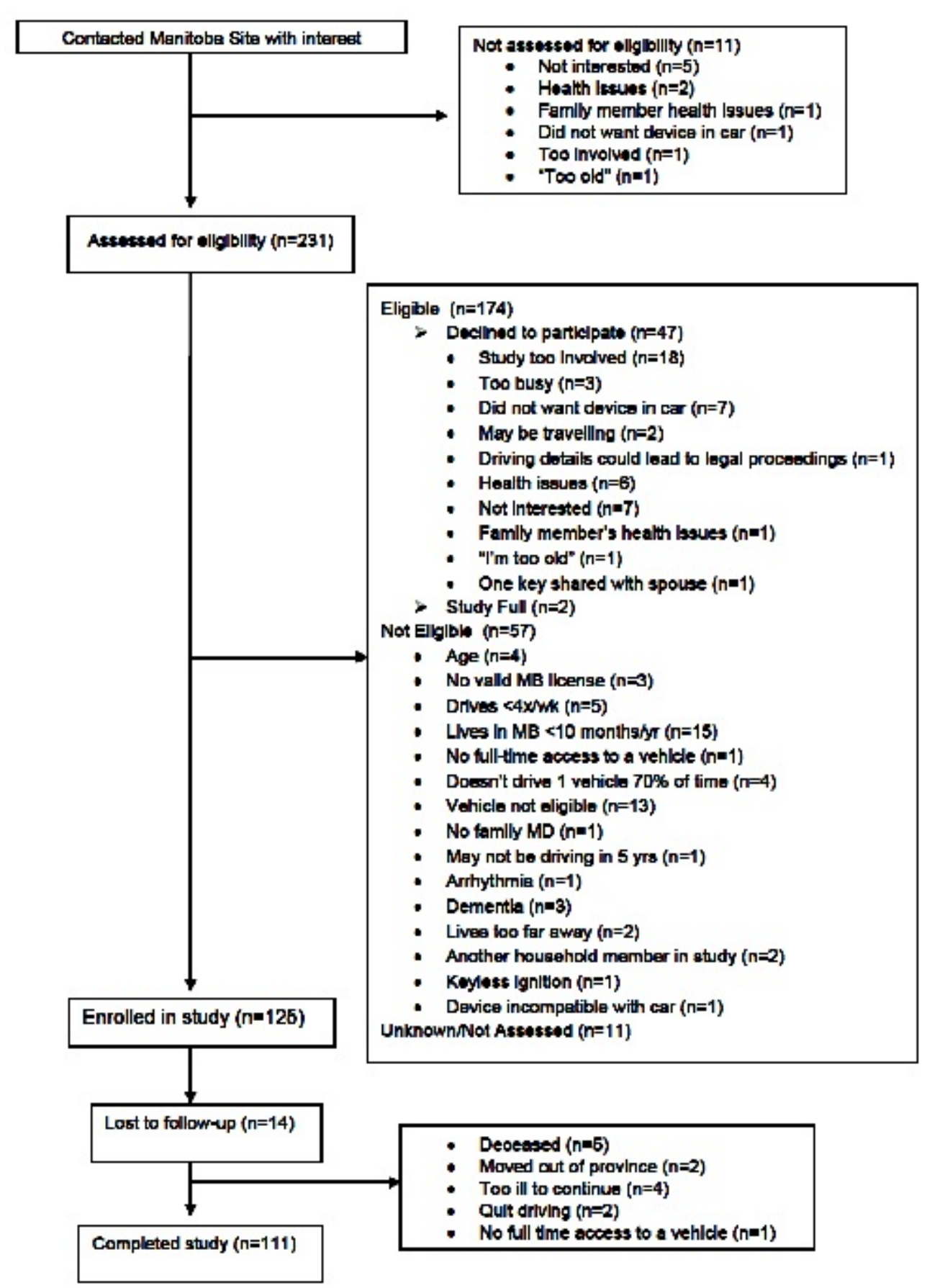

Figure 1. Recruitment, enrolment and retention of the participants in the study. 
Page 9 of 20

\subsection{Collision Data}

As mentioned above, the three forms of collision data collected were: driver abstracts, insurance claims, and self-reported information on collisions. Driver abstracts amongst other details (driver characteristics, type of license, any restrictions on their license, etc.) record at-fault collisions for drivers. On the abstract it is also indicated whether the collision involved property damage or any injuries. Initially (2009 to September 2011), only collisions that had an accompanying police report were recorded on the driver abstract by MPI. At that time, a collision that resulted in at least $\$ 1,000$ of property damage required a police report. However, the law in Manitoba changed in 2011 such that police reports only needed to be done when there were fatalities, hospitalization because of injuries, an unlicensed or unregistered driver, an unidentified vehicle (e.g., hit and run), an inability to get a driver's particulars, or impaired driving (MPI, 2017). Reporting to police also happened in instances of theft, attempted theft, or vandalism, although these claims were not relevant to this study. Since October 2011, all at-fault collisions, as deemed by MPI, regardless of the amount of property damage, were recorded on the driver abstract, if there was a pay out from MPI.

An insurance claim, for the purposes of this study, resulted from anyone involved reporting a collision to MPI. Details related to the claim included: date, time, location, amount of property damage incurred, were there any injuries, and some comments about the collision. In addition, liability as a percentage was also provided. Anything with a liability of $50 \%$ or more was deemed to be an at-fault collision by MPI. MPI adjusters determine liability in each case by getting statements from the driver(s) involved, witnesses, and sometimes passengers. They also compare the vehicle damage to the driver statements. They could even visit the collision scene, and review police reports, when available. 
Participants were also asked to self-report on any collisions they were in during the time they were enrolled in the study, regardless of who was at-fault. They could report a collision which occurred by contacting study staff at any time. They were also specifically asked about collisions when follow up appointments occurred, approximately every 4 months. The specific question about whether a collision occurred was: "Have you been involved in any collisions/accidents (including fender-benders) in the past four months?". If they answered "Yes", then a separate questionnaire was used to obtain details about the collision. Pertinent questions from the questionnaire included:
a. Where did this collision take place?
b. Was the collision reported to the police?
c. Extent of damage [participant vehicle as well as the other vehicle(s), if applicable]
d. Were there any persons (driver(s) or passengers) injured as a result of the collision?
e. Was an 'at fault' status determined? If yes, do you know who was considered at fault? (Other driver, you, unknown, other)

All forms of data were checked for concordance. In cases of differences between potentially similar collisions (e.g., date of collision) then other details from the claim or self-report were examined to ensure that the collision reported was the same.

\section{Results}

In total there were 68 participants who crashed during the followup period (based on self-report, the driver abstract, or an insurance claim). These participants were similar in characteristics to the Manitoba sample overall at enrollment. They ranged in age from 70 to $87(75.5 \pm 4.6)$ years, with $67.6 \%$ being men. 
Page 11 of 20

Across the four years, combining all data, there were 101 separate instances of collisions. Participants self-reported 92 collisions, there were 20 at-fault collisions reported on the driver abstract, and there were 70 insurance claims.

The 20 collisions on the driver abstract involved 20 different participants. Fourteen involved property damage, 3 involved injuries and 3 were with fixed objects. The incurred amount of the 20 collisions ranged from $\$ 601$ to $\$ 13,417$ (mean $=\$ 2,997.76, S D=\$ 3,226.92)$. Only 2 individuals did not report the collisions that were ultimately on their driver abstract. Both of these individuals did report other collisions. One participant who did not report a collision from their driver abstract may have been confused because of timing, because the collision occurred on the day of their assessment, and so they might have thought that they had reported it previously. There were also two individuals who reported the collision but did not indicate that they were at-fault. One said it was unknown, and the other person had not reported the collision to MPI at the time of their self-report.

For the 70 insurance claims, there were 51 participants involved. Thirty-six individuals had one claim, 12 had 2 claims, 2 had 3 claims and 1 individual had 4 claims. Of the 70 claims, 42 were at-fault -41 were determined to be $100 \%$ and the other one was $50 \%$. For those who were at-fault the incurred amount ranged from $\$ 0$ to $\$ 13,417$ (mean $=\$ 2,360.90, S D=\$ 2,742.30)$, and 23 were over $\$ 1,000$. Nine participants did not report a collision which was eventually associated with a claim. One claim was quite mysterious as there was no apparent damage to any vehicles, even though someone else reported that there was, and so it is not surprising that the participant did not report it. One participant had a garage door of a parkade come down on their vehicle so this might have not been interpreted as a collision to them. Similarly, two hit objects (one in a garage, another a pole), and another person hit a parked 
vehicle. As mentioned previously one person had a collision on the same day as a study appointment and so might have misjudged the timing (i.e., thought they had already reported it), and this also happened to another participant. Another participant actually had two collisions during a follow up period, but they only reported one collision. No one was incorrect with regards to the at-fault status, however, several were listed as 'unknown' or 'N/A' at the time of the self-report because a determination had not been made yet, or it had not been reported to MPI yet.

In terms of how many collisions made it to the driving abstract compared to insurance claims, there should have been 28 cases, based on the laws of the time. Prior to October 2011 this meant the damage was $\$ 1,000$ or more and the participant was at-fault. Starting in October 2011 , the collision just had to be at-fault, and have a pay-out of some amount. This means that $28.6 \%$ of at-fault collisions did not make it to the driver abstract that should have. There were 3 additional collisions with other vehicles (e.g., two vehicles were involved) that were reported to the insurer and were recorded as the participant being at-fault for the collision, but no payouts were made from the insurer, so likely that is why they were not recorded on the driver abstract.

Sixty-five participants were involved in the 92 self-reported collisions. Thirty-one self-reported collisions were not reported to MPI (30.7\% of the 101 total collisions). Many of these unclaimed collisions did not involve another vehicle $(\mathrm{n}=17)$ and were categorized (self-report) as being of 'no damage' or 'minor' damage. For those that did involve another vehicle, the self-reported damage to the other vehicle was 'no damage' or 'minor'. The participants, associated with these unclaimed self-reported collisions, were very similar in their age and gender distributions to the overall sample. 
Page 13 of 20

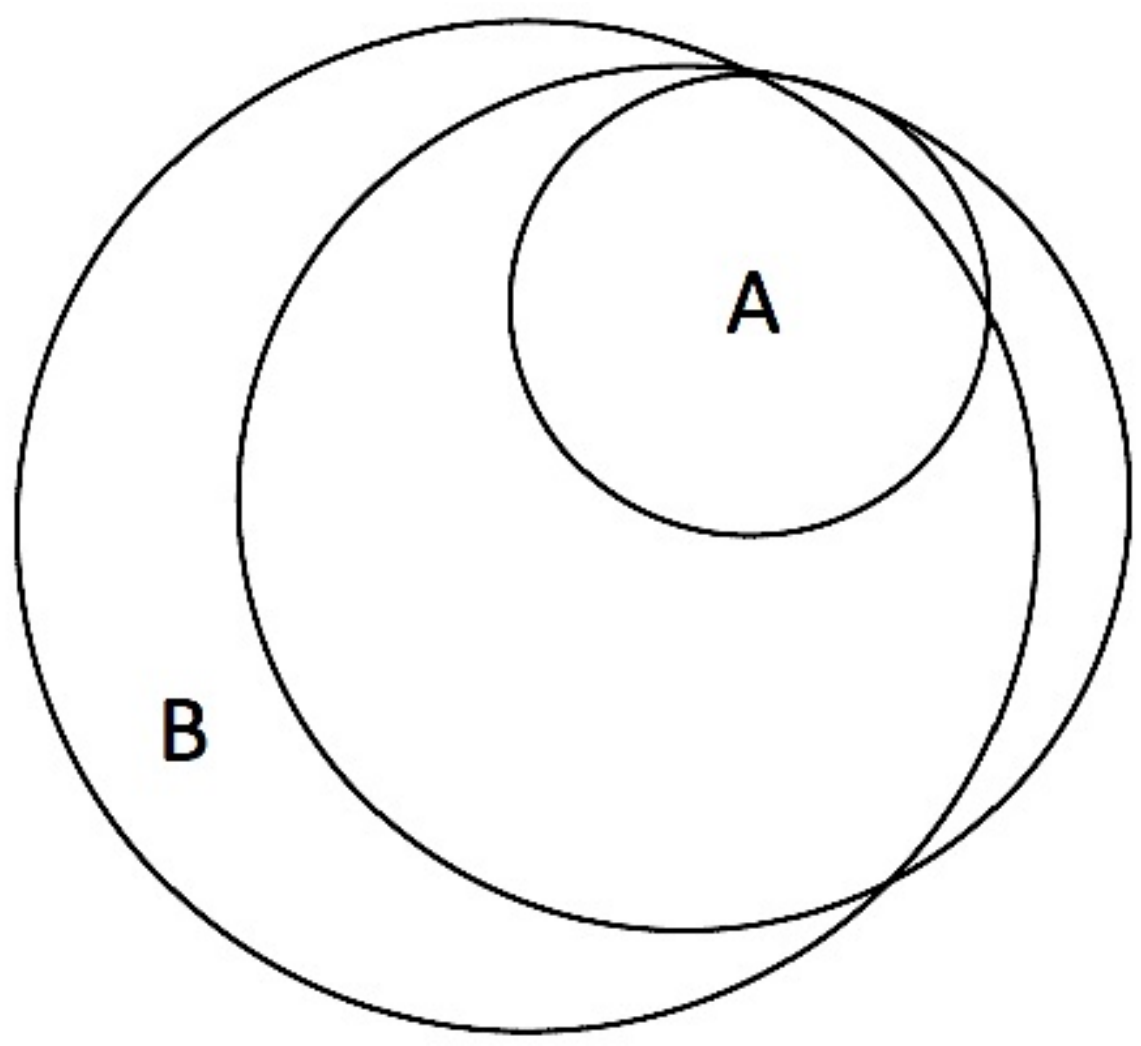

Figure 2. A Venn diagram of the types of collisions. The large circle represents self-reported collisions.

The medium size circle represents insurance claims. The smallest circle represents collisions on the official driver abstract (only at-fault collisions). The areas where there are overlapping circles represent collisions that were reported in multiple ways. For example, A represents collisions that were selfreported, part of an insurance claim and also on the driver abstract. The places where there is no overlap indicate collisions that were reported in only one way. The largest area of no overlap (B) is for collisions that were self-reported but were not part of the driver abstract or an insurance claim. 


\section{Discussion}

In this prospective study of older drivers, there were a number of discrepancies between the different collision data sources. The largest discrepancy was the number $(n=31)$ of self-reported collisions that were not reported to the licensing/insuring agency. In contrast, far fewer collisions that had become part of the official driver record, whether as an insurance claim $(n=9)$ or on the official driving abstract $(n=2)$, were not self-reported by the older drivers. Regardless of the source though, there appear to be missing collisions. Even between the insurance database and the official driver abstract, which were both managed by the same entity in this case, there were instances of missing at-fault collisions.

Previous research has indicated that more injury and damage information can be identified through insurance claims as compared to police reports (Mills et al., 2011). Our results concur with this finding. Although Mills et al. found that some research might not be affected by this missing data, they did suggest that when "evaluating absolute risk" of collisions this missing information becomes much more important.

While some researchers have questioned the validity of using self-reported collisions (af Wåhlberg and Dorn, 2015; McGwin et al., 1998) , the results here suggest that older drivers in a prospective trial reported almost 31\% more collisions than even made it on the insurance record. Marottoli et al. (1997) and Anstey et al. (2009) also found that older drivers retrospectively self-report more collisions than are part of the official record.

Reasons for self-reporting more collisions could be due to the extent of the damage related to the collision, and/or the fact that another driver was not involved. Also, there are obviously financial and 
legal implications for not reporting a collision. For example, costs for licensing/insuring could go up in the future, depending on the person's already existing driving record. It would be interesting to determine whether there are any age-related differences in self-reporting of collisions that are not claimed through insurance agencies, across the entire age spectrum, as this may affect our thinking about road safety. In this study, we did not ask participants why they did not report the collision, so we are not able to infer reasons. However, these participants were not different from the overall group in their age or gender distributions, so it does not appear that the oldest drivers, those in their 80 s for example, were more likely to avoid reporting. Future studies that examine the relationships between collisions and functional/medical status of older drivers could specifically study whether self-reported collisions that are not reported to insurance agencies are key indicators of medical fitness to drive, because, regardless of the associated damage costs, surely they give some suggestion of a driver's failure to completely manage their vehicle, and so could be used as a proxy for driver safety or performance.

In this study, although the rate of not-reporting collisions was low, there were instances whereby collisions that either became part of an insurance claim or were on the driver abstract were not reported to study staff. This could be due to confusion over timing of the collision relative to what was selfreported previously, or it could be due to memory or self-presentation issues (af Wahlberg and Dorn, 2015).

Finestone et al. (2011) also found discrepancies between self-reported collisions and those contained in the licensing authority record. They suggest that "the use of both government and self-reported data would ensure a more accurate picture of driving safety...." (pg 332). As mentioned above, others have 
found differences in risk factors or predictors of crash when they used different sources of information on crashes (Arthur et al., 2005; McGwin et al., 1998; Smith, 1976), so choosing the main outcome variable can be very important.

When using retrospective designs, particularly when asking about specific types of collisions (only policereported) over a long time period ( 5 years), it seems prudent to not use self-reported collisions, as per the recommendation of McGwin et al. (1998). However, the results of this prospective trial, and other studies with shorter retrospective designs (Anstey et al., 2009; Singletary et al., 2017), would suggest that self-reports might well be very useful.

The strengths of the current study are its longitudinal design, the multiple sources of collision data, the time period over which drivers were followed, and the fact that insurance claims were examined. Some weaknesses include the fact that there is no information on how much damage was incurred in selfreported collisions that had no insurance claims associated with them. This meant that we were not able to compare how insurance-reported and not reported collisions compared in terms of severity. It could be that they were very minor and less damaging collisions than those associated with an insurance claim, but we have no way of verifying this. We also have no way of independently attributing fault to these collisions, although many only involved one vehicle and so would imply that the participant was at-fault. Finally, because the participants knew we were also collecting data from the licensing authority/insurer this could have increased their rates of self-reporting collisions. However, this would not explain why they reported so many that were not part of official records. 
Page 17 of 20

In summary, in this longitudinal study of older drivers, self-reported collisions far outnumbered insurer or licensing authority records of collisions. The older drivers self-reported most collisions that were part of either official record, with a few exceptions. And even with official records tabulated by the same agency, there were discrepancies in the at-fault collisions that were part of the older drivers' records. Therefore, researchers need to be cautious when using any source of collision data when trying to assess who is most likely to crash.

Funding source

This studywas funded by a TeamGrant fromthe Canadian Institutesof Health Research (CIHR) entitled “The CIHR Team in Driving in Older Persons (Candrive II) Research Program” (grant 90429; www.candrive.ca). The sponsor had no role in the research or the manuscript. 


\section{References}

af Wåhlberg, A.E., Dorn, L., 2015. How reliable are self-report measures of mileage, violations and crashes. Safety Science 76, 67-73.

Anstey, K.J., Wood, J., Caldwell, H., Kerr, G., Lord, S.R., 2009. Comparison of self-reported crashes, state crash records and on-road driving assessment in a population-based sample of drivers aged 69-95 years. Traffic Injury Prevention 10, 84-90.

Arthur, W. Jr., Bell, S.T., Edwards, B.D., Day, E.A., Tubre, T.C., Tubre, A.H., 2005. Convergence of selfreport and archival crash involvement data: a two-year longitudinal follow-up. Human Factors 47, 303313.

Ball, K., Owsley, C., Sloane, M.E., Roenker, D.L., Bruni, J.R., 1993. Visual attention problems as a predictor of vehicle crashes in older drivers. Investigative Ophthalmology \& Visual Science 34, 31103123.

Finestone, H.M., Guo, M., O’Hara, P., Greene-Finestone, L., Marshall, S.C., Hunt, L., Jessup, A. Biggs, J. 2011. Department of Transportation vs self-reported data on motor vehicle collisions and driving convictions for stroke survivors: do they agree? Traffic Injury Prevention 12, 327-332.

Gagnon, S., Marshall, S.C., Kadulina, Y., Stinchcombe, A., Bédard, M., Gélinas, I., Man-Son-Hing, M., Mazer, B., Naglie, G., Porter, M.M., Rapoport, M., Tuokko, H., Vrklnan, B. on Behalf of the Candrive 
Research Team, 2016. Assessing the Representativeness of a Convenience Sample of Older Drivers: A Comparison between the CIHR Candrive Cohort Study Sample and the Household Population of Canadians with Valid Driver's Licenses. Canadian Journal on Aging, 35(S1), 99 - 109.

Manitoba Public Insurance (MPI). Notifying police after an accident. https://www.mpi.mb.ca/en/Claims/Vehicle/Reporting/Pages/notify-police.aspx, accessed July 5, 2017.

Marottoli, R.A., Cooney, L.M. Jr., Tinetti, M.E., 1997. Self-report versus state records for identifying crashes among older drivers. Journals of Gerontology: Medical Sciences 52A, M184-M187.

Marshall, S.C., Man-Son-Hing, M., Bédard, M., Porter, M.M., Charlton, J., Gagnon, S., Gélinas, I., Koppel, S., Korner-Bitensky, N., Langford, J., Mazer, B., Myers, A., Naglie, G., Polgar, J., Rapoport, M., Tuokko, H., Vrkljan, B., Woolnough A., 2013. Protocol for Candrive II/Ozcandrive, a multicentre prospective older driver cohort study. Accident Analysis and Prevention, S61, 245-252.

McGuire, F.I., 1973. The nature of bias in official accident and violation records. Journal of Applied Psychology 57, 300-305.

McGwin, G. Jr., Owsley, C., Ball, K., 1998. Identifying crash involvement among older drivers: agreement between self-report and state records. Accident Analysis and Prevention 30, 781-791. 
Page 20 of 20

Mills, B.N., Andrey, J., Hambly, D., 2011. Analysis of precipitation-related motor vehicle collision and injury risk using insurance and police record information for Winnipeg, Canada. Journal of Safety Research 42, 383-390.

Owsley, C., Ball, K., Sloane, M.E., Roenker, D.L., Bruni, J.R., 1991. Visual/cognitive correlates of vehicle accidents in older drivers. Psychology and Aging 6, 403-415.

Singletary, B.A., Do, A.N., Donnelly, J.P., Huisingh, C., Mefford, M.T., Modi, R., Mondesir, F.L., Ye, Y., Owsley, C., McGwin, G., 2017. Self-reported vs state-recorded motor vehicle collisions among older community dwelling individuals. Accident Analysis and Prevention 101, 22-27.

Smith, D.I., 1976. Official driver records and self-reports as sources of accident and conviction data for research purposes. Accident Analysis and Prevention 8, 207-211.

Zylman, R., 1972. Drivers' records: are they a valid measure of driving behavior? Accident Analysis and Prevention 4, 333-349. 